\title{
Light scalar mesons and two-kaon correlation functions
}

\author{
N. N. Achasov ${ }^{1}$ and A. V. Kiselev ${ }^{1,2}$ \\ ${ }^{1}$ Laboratory of Theoretical Physics, Sobolev Institute for Mathematics, 630090 Novosibirsk, Russia \\ ${ }^{2}$ Novosibirsk State University, 630090 Novosibirsk, Russia
}

(Received 23 November 2017; revised manuscript received 8 January 2018; published 16 February 2018)

\begin{abstract}
It is shown that the recent data on the $K_{S}^{0} K^{+}$correlation in $\mathrm{Pb}-\mathrm{Pb}$ interactions agree with the data on the $\gamma \gamma \rightarrow \eta \pi^{0}$ and $\phi \rightarrow \eta \pi^{0} \gamma$ reactions and support the four-quark model of the $a_{0}(980)$ meson. It is shown that the data does not contradict the validity of the Gaussian assumption. The study of two-kaon correlations could provide more information about light scalar mesons after increasing the accuracy of the experimental and theoretical descriptions.
\end{abstract}

DOI: 10.1103/PhysRevD.97.036015

\section{INTRODUCTION}

The $a_{0}(980)$ and $f_{0}(980)$ mesons are well-established parts of the proposed light scalar meson nonet [1]. From the beginning, the $a_{0}(980)$ and $f_{0}(980)$ mesons became one of the central problems of nonperturbative QCD, as they are important for understanding the way chiral symmetry is realized in the low-energy region and, consequently, for understanding confinement. Many experimental and theoretical papers have been devoted to this subject.

There is much evidence that supports the four-quark model of light scalar mesons [2].

First, the suppression of the $a_{0}(980)$ and $f_{0}(980)$ resonances in the $\gamma \gamma \rightarrow \eta \pi^{0}$ and $\gamma \gamma \rightarrow \pi \pi$ reactions, respectively, was predicted in 1982 [3], $\Gamma_{a_{0} \gamma \gamma} \approx \Gamma_{f_{0} \gamma \gamma} \approx 0.27 \mathrm{keV}$, and confirmed by experiment [1]. The elucidation of the mechanisms of the $\sigma(600), f_{0}(980)$, and $a_{0}(980)$ resonance production in $\gamma \gamma$ collisions confirmed their fourquark structure $[4,5]$. Light scalar mesons are produced in $\gamma \gamma$ collisions mainly via rescatterings, that is, via the fourquark transitions. As for $a_{2}(1320)$ and $f_{2}(1270)$ (the wellknown $q \bar{q}$ states), they are produced mainly via the two-quark transitions (direct couplings with $\gamma \gamma$ ).

Second, the argument in favor of the four-quark nature of $a_{0}(980)$ and $f_{0}(980)$ is the fact that the $\phi(1020) \rightarrow a_{0} \gamma$ and $\phi(1020) \rightarrow f_{0} \gamma$ decays go through the kaon loop: $\phi \rightarrow K^{+} K^{-} \rightarrow a_{0} \gamma, \phi \rightarrow K^{+} K^{-} \rightarrow f_{0} \gamma$, i.e., via the fourquark transition [6-10]. The kaon-loop model was suggested in Ref. [9] and confirmed by experiment ten years later [11-13].

Published by the American Physical Society under the terms of the Creative Commons Attribution 4.0 International license. Further distribution of this work must maintain attribution to the author(s) and the published article's title, journal citation, and DOI. Funded by SCOAP ${ }^{3}$.
It was shown in Ref. [6] that the production of $a_{0}(980)$ and $f_{0}(980)$ in $\phi \rightarrow a_{0} \gamma \rightarrow \eta \pi^{0} \gamma$ and $\phi \rightarrow f_{0} \gamma \rightarrow \pi^{0} \pi^{0} \gamma$ decays is caused by the four-quark transitions, resulting in strong restrictions on the large- $N_{C}$ expansions of the decay amplitudes. The analysis showed that these constraints give new evidence in favor of the four-quark nature of the $a_{0}(980)$ and $f_{0}(980)$ mesons.

Third, in Refs. $[14,15]$ it was shown that the description of the $\phi \rightarrow K^{+} K^{-} \rightarrow \gamma a_{0}(980) / f_{0}(980)$ decays requires virtual momenta of $K(\bar{K})$ greater than $2 \mathrm{GeV}$, while in the case of loose molecules with a binding energy about $20 \mathrm{MeV}$, they would have to be about $100 \mathrm{MeV}$. Besides, it should be noted that the production of scalar mesons in the pion-nucleon collisions with large momentum transfers also points to their compactness [16].

Fourth, the data on semileptonic $D_{s}^{+} \rightarrow s \bar{s} e^{+} \nu \rightarrow$ $\left[\sigma(600)+f_{0}(980)\right] e^{+} \nu \rightarrow \pi^{+} \pi^{-} e^{+} \nu$ decays are also in favor of the four-quark nature of $\sigma(600)$ and $f_{0}(980)$ [17]. Unfortunately, at the moment the statistics is rather poor, and thus new high-statistics data are highly desirable. No less interesting is the study of semileptonic decays of $D^{0}$ and $D^{+}$mesons $-D^{0} \rightarrow d \bar{u} e^{+} \nu \rightarrow a_{0}^{-} e^{+} \nu \rightarrow \pi^{-} \eta e^{+} \nu$, $D^{+} \rightarrow d \bar{d} e^{+} \nu \rightarrow a_{0}^{0} e^{+} \nu \rightarrow \pi^{0} \eta e^{+} \nu$ (or the charged-conjugated ones) and $D^{+} \rightarrow d \bar{d} e^{+} \nu \rightarrow\left[\sigma(600)+f_{0}(980)\right]$ $e^{+} \nu \rightarrow \pi^{+} \pi^{-} e^{+} \nu$-which have not been investigated yet $[17,18]$. It is very tempting to study light scalar mesons in semileptonic decays of $B$ mesons [18]: $B^{0} \rightarrow d \bar{u} e^{+} \nu \rightarrow$ $a_{0}^{-} e^{+} \nu \rightarrow \pi^{-} \eta e^{+} \nu, B^{+} \rightarrow u \bar{u} e^{+} \nu \rightarrow a_{0}^{0} e^{+} \nu \rightarrow \pi^{0} \eta e^{+} \nu, B^{+} \rightarrow$ $u \bar{u} e^{+} \nu \rightarrow\left[\sigma(600)+f_{0}(980)\right] e^{+} \nu \rightarrow \pi^{+} \pi^{-} e^{+} \nu$.

It was also shown in Refs. $[19,20]$ that the linear $S_{L}(2) \times$ $S_{R}(2) \sigma$ model [21] reflects all of the main features of lowenergy $\pi \pi \rightarrow \pi \pi$ and $\gamma \gamma \rightarrow \pi \pi$ reactions and agrees with the four-quark nature of light scalar mesons. This allowed for the development of a phenomenological model with the right analytical properties in the complex $s$ plane that took into account the linear $\sigma$ model and the background [22]. 
This background has a left cut inspired by crossing symmetry, and the resulting amplitude agrees with results obtained using the chiral expansion, dispersion relations, and the Roy equation [23], and with the four-quark nature of the $\sigma(600)$ and $f_{0}(980)$ mesons as well.

Recently, the ALICE Collaboration's investigation of $K_{S}^{0} K^{ \pm}$correlation [24] determined that $a_{0}(980)$ is a fourquark state. This conclusion was made on the basis that masses and coupling constants obtained in the four-quarkbased scenario from the data on $\phi \rightarrow \eta \pi^{0} \gamma$ and $\phi \rightarrow \pi^{0} \pi^{0} \gamma$ decays $[7,8,13]$ accurately describe the data on twokaon correlations, in contradistinction to other sets of parameters [25].

Statistically significant data on two-kaon correlations appeared recently. In 2006, the STAR Collaboration presented data on $K_{S}^{0} K_{S}^{0}$ correlation in $\mathrm{Au}-\mathrm{Au}$ interactions [26]. Both $a_{0}^{0}(980)$ and $f_{0}(980)$ are created in the process.

Recently, the ALICE Collaboration published data on $K_{S}^{0} K^{ \pm}$correlations in $\mathrm{Pb}-\mathrm{Pb}$ interactions [24], and $a_{0}^{ \pm}(980)$ is created in these reactions.

In 2015, the authors of [27] presented an analysis on the Belle data on the $\gamma \gamma \rightarrow \eta \pi^{0}$ reaction together with KLOE data on the decay $\phi \rightarrow \eta \pi^{0} \gamma$. Here, we present a new analysis which additionally includes the ALICE data on $K_{S}^{0} K^{+}$correlation [24].

We justify the $a_{0}(980)$ four-quark nature on a higher level than that in Ref. [24]: the set of different data (the Belle data on $\gamma \gamma \rightarrow \eta \pi^{0}$, the KLOE data on $\phi \rightarrow \eta \pi^{0} \gamma$, and the ALICE data on two-kaon correlation) is simultaneously described in a scenario based on the four-quark model [2]. In this scenario the coupling constants obey (or almost obey) the relations [9]

$$
\begin{aligned}
g_{a_{0} \eta \pi^{0}} & =\sqrt{2} \sin \left(\theta_{p}+\theta_{q}\right) g_{a_{0} K^{+} K^{-}} \\
& =(0.85 \div 0.98) g_{a_{0} K^{+} K^{-}}, \\
g_{a_{0} \eta^{\prime} \pi^{0}} & =-\sqrt{2} \cos \left(\theta_{p}+\theta_{q}\right) g_{a_{0} K^{+} K^{-}} \\
& =-(1.13 \div 1.02) g_{a_{0} K^{+} K^{-}},
\end{aligned}
$$

and the coupling to the $\gamma \gamma$ channel is small. Here $g_{a_{0} \eta \pi^{0}}=$ $0.85 g_{a_{0} K^{+} K^{-}}$and $g_{a_{0} \eta^{\prime} \pi^{0}}=-1.13 g_{a_{0} K^{+} K^{-}}$for $\theta_{p}=-18^{\circ}$ and $g_{a_{0} \eta \pi^{0}}=0.98 g_{a_{0} K^{+} K^{-}}$and $g_{a_{0} \eta^{\prime} \pi^{0}}=-1.02 g_{a_{0} K^{+} K^{-}}$for $\theta_{p}=-11^{\circ}$. The $\theta_{q}=54.74^{\circ}$.

Our description takes into account the $a_{0}^{\prime}$ meson and uses one-loop scalar propagators with good analytical properties; see Sec. II.

The approach is based on Ref. [28], which in turn was based on the assumption of an ideal chaotic Gaussian source, which requires that the correlation strength $\lambda$ be equal to unity; for details, see Ref. [29]. We show that the data is described well with $\lambda=1$, what didn't manage to be made in Ref. [24].

Note that we do not use the STAR and ALICE data on the correlation of identical kaons $[26,30,31]$ because in the charged case $a_{0}(980)$ is not created, and the neutral case deals both with isospin $I=0$ and $I=1$, i.e., a similar simultaneous analysis would require taking into account $f_{0}(980), f_{2}(1270)$, and $\sigma(600)$ and the reactions $\gamma \gamma \rightarrow \pi^{0} \pi^{0}, \phi \rightarrow \pi^{0} \pi^{0} \gamma$, and $\pi \pi \rightarrow \pi \pi$. This is a rather complicated problem, and we hope to return to it in the future.

\section{FORMALISM AND RESULTS}

Let us briefly consider the formalism used in Ref. [24], which is based on that in Ref. [28]. The scattering amplitude is [Eq. (6) of Ref. [24]]

$$
f\left(k^{*}\right)=\frac{\gamma_{a_{0} \rightarrow K \bar{K}}}{m_{a_{0}}^{2}-s-i\left(\gamma_{a_{0} \rightarrow K \bar{K}} k^{*}+\gamma_{a_{0} \rightarrow \pi \eta} k_{\pi \eta}\right)} .
$$

Here the denominator is the inverse propagator of $a_{0}^{+}$in a Flatté-like form [32], $s$ is the invariant two-kaon mass squared, $k^{*}$ is the kaon momentum in the kaon pair rest frame,

$$
k^{*}=\frac{\sqrt{\left(s-\left(m_{K_{S}^{0}}-m_{K^{+}}\right)^{2}\right)\left(s-\left(m_{K_{S}^{0}}+m_{K^{+}}\right)^{2}\right)}}{2 \sqrt{s}},
$$

and $k_{\pi \eta}$ is the corresponding $\pi \eta$ momentum.

The correlation $C\left(k^{*}\right)$ is [Eq. (9) of Ref. [24]]

$$
\begin{aligned}
C\left(k^{*}\right)= & 1+\frac{\lambda}{2}\left(\frac{1}{2}\left|\frac{f\left(k^{*}\right)}{R}\right|^{2}+2 \frac{\operatorname{Ref}\left(k^{*}\right)}{\sqrt{\pi} R} F_{1}\left(2 k^{*} R\right)\right. \\
& \left.-\frac{\operatorname{Im} f\left(k^{*}\right)}{R} F_{2}\left(2 k^{*} R\right)\right),
\end{aligned}
$$

where $R$ is the radius parameter from the spherical Gaussian source distribution, $\lambda$ is the correlation strength, and

$$
F_{1}(z)=\frac{e^{-z^{2}}}{z} \int_{0}^{z} e^{x^{2}} d x ; \quad F_{2}(z)=\frac{1-e^{-z^{2}}}{z} .
$$

The Flatté propagator is not adequate for studying $f_{0}(980)$ and $a_{0}(980)$; see Refs. [10,14,33-35]. As in Ref. [27], we use one-loop propagators and take into account the $a_{0}^{\prime+}$ meson, so Eq. (4) is modified:

$$
f\left(k^{*}\right)=\frac{2}{\sqrt{s}} \sum_{S, S^{\prime}} \frac{g_{S K_{S}^{0} K^{+}} G_{S S^{\prime}}^{-1} g_{S^{\prime} K_{S}^{0} K^{+}}}{16 \pi},
$$

where $S, S^{\prime}=a_{0}^{+}, a_{0}^{\prime+}$, and the constants $g_{S K_{S}^{0} K^{+}}=$ $-g_{S K_{L}^{0} K^{+}}=g_{S K^{+} K^{-}}$. The matrix of the inverse propagators is

$$
G_{S S^{\prime}} \equiv G_{S S^{\prime}}(m)=\left(\begin{array}{cc}
D_{a_{0}^{\prime}}(m) & -\prod_{a_{0}^{\prime} a_{0}}(m) \\
-\Pi_{a_{0}^{\prime} a_{0}}(m) & D_{a_{0}}(m)
\end{array}\right),
$$




$$
\Pi_{a_{0}^{\prime} a_{0}}(m)=\sum_{a, b} \frac{g_{a_{0}^{\prime} a b}}{g_{a_{0} a b}} \Pi_{a_{0}}^{a b}(m)+C_{a_{0}^{\prime} a_{0}},
$$

where $m=\sqrt{s}$, and the constant $C_{a_{0}^{\prime} a_{0}}$ incorporates the subtraction constant for the transition $a_{0}(980) \rightarrow\left(0^{-} 0^{-}\right) \rightarrow$ $a_{0}^{\prime}$ and effectively takes into account the contributions of multiparticle intermediate states to the $a_{0} \leftrightarrow a_{0}^{\prime}$ transition. The inverse propagator of the scalar meson $S[9,10,27,36]$ is

$D_{S}(m)=m_{S}^{2}-m^{2}+\sum_{a b}\left[\operatorname{Re} \Pi_{S}^{a b}\left(m_{S}^{2}\right)-\Pi_{S}^{a b}\left(m^{2}\right)\right]$,

where $\sum_{a b}\left[\operatorname{Re} \Pi_{S}^{a b}\left(m_{S}^{2}\right)-\Pi_{S}^{a b}\left(m^{2}\right)\right]=\operatorname{Re} \Pi_{S}\left(m_{S}^{2}\right)-\Pi_{S}\left(m^{2}\right)$ takes into account the finite-width corrections of the resonance, which are the one-loop contributions to the selfenergy of the $S$ resonance from the two-particle intermediate $a b$ states. We take into account the intermediate states $\eta \pi^{+}$, $K \bar{K}$, and $\eta^{\prime} \pi^{+}$in the $a_{0}^{+}(980)$ and $a_{0}^{\prime+}$ propagators:

$$
\Pi_{S}=\Pi_{S}^{\eta \pi^{+}}+\Pi_{S}^{K_{S}^{0} K^{+}}+\Pi_{S}^{K_{L}^{0} K^{+}}+\Pi_{S}^{\eta^{\prime} \pi^{+}} .
$$

The forms of $\Pi_{S}^{a b}(m)$ are expressed in Appendix A.

Equipped with these formulas, we fit the "previous" data (i.e., the data on $\gamma \gamma \rightarrow \eta \pi^{0}$ [37] and $\phi \rightarrow \eta \pi^{0} \gamma$ [13] reactions) as in Ref. [27] simultaneously with the ALICE data on $K_{S}^{0} K^{+}$correlation (29 points from the upper-left panel in Fig. 2 of Ref. [24]). Only statistical errors are taken into account.

Unfortunately, the ALICE Collaboration did not publish the data in the form of a table, with statistical, systematic, and total errors for combined $K_{S}^{0} K^{+}$and $K_{S}^{0} K^{-}$data sets. For safety, we neglect systematic error and do not fit the data on $K_{S}^{0} K^{-}$(the data sets are consistent).
We perform four analogs of Fit 1 of Ref. [27]; see Table I and Fig. 2. Parameters that are not mentioned above are in Table II of Appendix B. To fit the "previous" data we use the same $\chi^{2}$ functions with the same restrictions, including fixing the $a_{0}^{\prime}$ mass at $1400 \mathrm{MeV}$ and terms that guarantee being close to the four-quark model relations (1); for details, see Ref. [27]. The $\chi_{\text {corr }}^{2}$ in Table I is the usual $\chi^{2}$ function built on the $K_{S}^{0} K^{+}$correlation data.

In Table I, Fit 1 is for free $\lambda$ and $R$, and Fit 2 is for $\lambda=1$. One can see that the quality of Fit 2 is also good so the data does not contradict $\lambda$ being equal to unity.

Fits 3 and 4 are for the parameters of Fit 1 of Ref. [27], with free $\lambda$ and $R$ and with $\lambda=1$, respectively. One can see that Fit 3 describes the data quite well, while Fit 4 does not describe the data on correlation with a perfect $\chi^{2}$, though the description is not very bad since errors are small and systematic errors are neglected.

The difference in the $a_{0}$ features between Fits 1,2 and Fits 3,4 (with the "old" parameters) is rather small: the $a_{0}^{\prime}$ features are more fluid, as was observed in Ref. [27]. The description of "previous" data for Fit 1 is shown in Fig. 1: it is close to that in Ref. [27]. The correlation is shown in Fig. 2.

Analogs of other fits from Ref. [27] could be obtained in the same way.

As in Ref. [27], we do not calculate errors of the parameters. In our case, the minimized function has more than one minimum, for example, one with $\lambda=0.53$ (Fit 1) and another with $\lambda=0.66$ and $m_{a_{0}}=1012 \mathrm{MeV}$. The last value exceeds the usually obtained ones, but is also not excluded. The values of the minimized function differ by less than 1 in these minima, while for $\lambda$ in the intermediate region $0.53-0.66$ the deviation from the minimum values is greater than 1 .

\begin{tabular}{|c|c|c|c|c|}
\hline Fit & 1 & 2 & 3 & 4 \\
\hline$m_{a_{0}}, \mathrm{MeV}$ & 995.1 & 1003 & 993.9 & 993.9 \\
\hline$g_{a_{0} K^{+} K^{-}}, \mathrm{GeV}$ & 2.70 & 2.73 & 2.75 & 2.75 \\
\hline$g_{a_{0} \eta \pi}, \mathrm{GeV}$ & 2.85 & 2.95 & 2.74 & 2.74 \\
\hline$g_{a_{0} \eta^{\prime} \pi}, \mathrm{GeV}$ & -2.79 & -2.81 & -2.86 & -2.86 \\
\hline$m_{a_{0}^{\prime}}, \mathrm{MeV}$ & 1400 & 1400 & 1400 & 1400 \\
\hline$g_{a_{0}^{\prime} K^{+} K^{-}}, \mathrm{GeV}$ & 0.87 & 1.04 & 1.63 & 1.63 \\
\hline$g_{a_{0}^{\prime} \eta \pi}, \mathrm{GeV}$ & -2.33 & -2.72 & -3.12 & -3.12 \\
\hline$g_{a_{0}^{\prime} \eta^{\prime} \pi}, \mathrm{GeV}$ & -6.73 & -6.56 & -4.75 & -4.75 \\
\hline$C_{a_{0} a_{0}^{\prime}}, \mathrm{GeV}^{2}$ & 0.146 & 0.133 & 0.021 & 0.021 \\
\hline$\lambda$ & 0.53 & 1 & 0.73 & 1 \\
\hline$R, \mathrm{fm}$ & 5.0 & 6.7 & 5.6 & 6.8 \\
\hline$\chi_{\gamma \gamma}^{2} / 36$ points & 13.1 & 19.0 & 12.4 & 12.4 \\
\hline$\chi_{s p}^{2} / 24$ points & 24.7 & 25.6 & 24.5 & 24.5 \\
\hline$\chi_{\text {corr }}^{2} / 29$ points & 19.0 & 28.2 & 24.8 & 40.4 \\
\hline$\left(\chi_{\gamma \gamma}^{2}+\chi_{s p}^{2}+\chi_{\text {corr }}^{2}\right) /$ n.d.f. & $56.9 / 73$ & $72.8 / 74$ & $61.6 / 73$ & $77.2 / 74$ \\
\hline
\end{tabular}

TABLE I. Properties of the resonances and the description quality. 


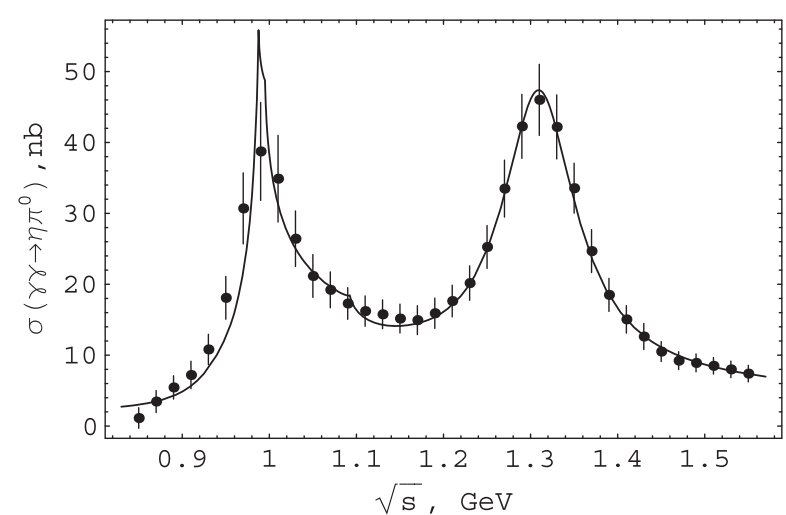

(a)

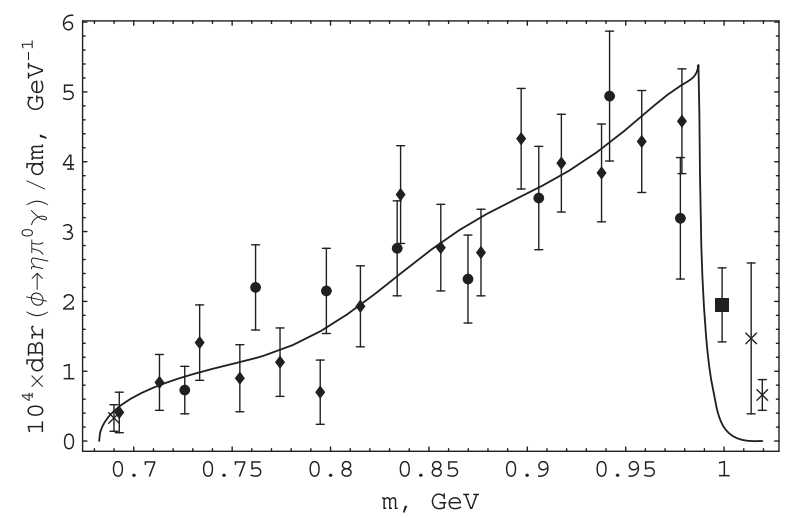

(b)

FIG. 1. (a) The $\gamma \gamma \rightarrow \eta \pi^{0}$ cross section: the curve is Fit 1, and the data points are from Belle [37]. Note that the Belle data represent the averaged cross section (each bin is $20 \mathrm{MeV}$ ). (b) Plot of the Fit 1 curve and the KLOE data (points) [13] on the $\phi \rightarrow \eta \pi^{0} \gamma$ decay; $m$ is the invariant $\eta \pi^{0}$ mass. Cross points are omitted in the fitting.

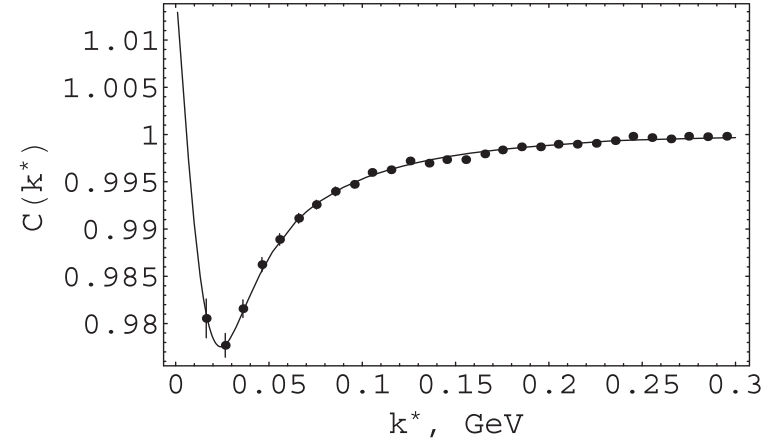

FIG. 2. $K_{S}^{0} K^{+}$correlation: the solid line corresponds to Fit 1, and the points are experimental data [24].

In Ref. [24] the obtained values of $\lambda$ were much less than 1 and not far from $\lambda=0.53$ in Fit 1 . We are able to obtain Fit 2 with $\lambda=1$ primarily because of the presence of $a_{0}^{\prime}$ and the fact that we vary the $a_{0}$ parameters. $a_{0}^{\prime}$ gives a notable contribution to the correlation: its removal raises $\chi_{\text {corr }}^{2}$ from 19 to 57 in Fit 1 and from 28 to 107 in Fit 2, while the other parameters remain the same. Also, we use a better propagator for the scalar particles.

Fits 1-3 show that the whole set of experimental data could be described in the four-quark model of $a_{0}(980)$. Moreover, the results of the previous analysis are well consistent with the data on correlation.

The predictive power of the data on correlation should increase a lot following advances in the description of the kaon generation process. Now we have two additional degrees of freedom $(R$ and $\lambda)$, and Fits 1-4 show that even if we only fix $\lambda$, the data become much more "strict".

Note that Eq. (4) is not a precise formula. Here $\lambda$ is an effective parameter that takes into account the nonGaussian distribution of the kaon source, etc. If the distribution is severely non-Gaussian, Eq. (4) should be completely modified: it is not enough to just introduce $\lambda$.
In Fits 1 and 3 and Ref. [24], the obtained values of $\lambda$ were not close to 1 ( $\approx 0.6$ in Ref. [24]). This raises the question of the self-consistency of the results (however, it could be explained by other effects; see Ref. [29]). As far as we understand, it is not easy to achieve progress in this field.

\section{CONCLUSION}

It was shown that the ALICE data on $K_{S}^{0} K^{+}$correlation could be described simultaneously with the Belle data on $\gamma \gamma \rightarrow \eta \pi^{0}$ and the KLOE data on $\phi \rightarrow \eta \pi^{0} \gamma$ in a scenario based on the four-quark model.

Fit 2 shows that the data could be well described with the correlation strength $\lambda$ equal to unity, as it should be for an ideal chaotic Gaussian source. However, we emphasize that the current experimental data does not allow us to make strict conclusions on $\lambda$.

\section{ACKNOWLEDGMENTS}

The present work is partially supported by the Russian Foundation for Basic Research Grant No. 16-02-00065 and the Presidium of the Russian Academy of Sciences Project Grant No. 0314-2015-0011.

\section{APPENDIX A: POLARIZATION OPERATORS}

For pseudoscalar mesons $a, b$ and $m_{a} \geq m_{b}, m \geq m_{+}$, one has

$$
\begin{aligned}
\Pi_{S}^{a b}\left(m^{2}\right)= & \frac{g_{\text {Sab }}^{2}}{16 \pi}\left[\frac{m_{+} m_{-}}{\pi m^{2}} \ln \frac{m_{b}}{m_{a}}\right. \\
& \left.+\rho_{a b}\left(i+\frac{1}{\pi} \ln \frac{\sqrt{m^{2}-m_{-}^{2}}-\sqrt{m^{2}-m_{+}^{2}}}{\sqrt{m^{2}-m_{-}^{2}}+\sqrt{m^{2}-m_{+}^{2}}}\right)\right],
\end{aligned}
$$


where $\rho_{a b}(s)=2 p_{a b}(s) / \sqrt{s}=\sqrt{\left(1-m_{+}^{2} / s\right)\left(1-m_{-}^{2} / s\right)}$, and $m_{ \pm}=m_{a} \pm m_{b}$.

For $m_{-} \leq m<m_{+}$,

$$
\Pi_{S}^{a b}\left(m^{2}\right)=\frac{g_{S a b}^{2}}{16 \pi}\left[\frac{m_{+} m_{-}}{\pi m^{2}} \ln \frac{m_{b}}{m_{a}}-\left|\rho_{a b}(m)\right|+\frac{2}{\pi}\left|\rho_{a b}(m)\right| \arctan \frac{\sqrt{m_{+}^{2}-m^{2}}}{\sqrt{m^{2}-m_{-}^{2}}}\right],
$$

and for $m<m_{-}$,

$$
\Pi_{S}^{a b}\left(m^{2}\right)=\frac{g_{S a b}^{2}}{16 \pi}\left[\frac{m_{+} m_{-}}{\pi m^{2}} \ln \frac{m_{b}}{m_{a}}-\frac{1}{\pi} \rho_{a b}(m) \ln \frac{\sqrt{m_{+}^{2}-m^{2}}-\sqrt{m_{-}^{2}-m^{2}}}{\sqrt{m_{+}^{2}-m^{2}}+\sqrt{m_{-}^{2}-m^{2}}}\right] .
$$

The constants $g_{\text {Sab }}$ are related to the width as

$$
\Gamma_{S}(m)=\sum_{a b} \Gamma(S \rightarrow a b, m)=\sum_{a b} \frac{g_{\mathrm{Sab}}^{2}}{16 \pi m} \rho_{a b}(m) .
$$

\section{APPENDIX B: OTHER PARAMETERS}

For completeness, we show parameters that are not described above in Table II. One can find all of the details in Ref. [27].

TABLE II. Parameters not mentioned in Table I.

\begin{tabular}{lcccc}
\hline \hline Fit & 1 & 2 & 3 & 4 \\
\hline$g_{a_{0} \gamma \gamma}^{(0)}, 10^{-3} \mathrm{GeV}^{-1}$ & 1.8 & 1.8 & 1.8 & 1.8 \\
$g_{a_{0}^{\prime} \gamma \gamma}, 10^{-3} \mathrm{GeV}^{-1}$ & 8.53 & 7.73 & 5.5 & 5.5 \\
$c_{0}$ & 8.8 & 8.1 & 10.3 & 10.3 \\
$c_{1}, \mathrm{GeV}^{-2}$ & -20.1 & -18.4 & -24.2 & -24.2 \\
$c_{2}, \mathrm{GeV}^{-4}$ & -0.001 & -0.002 & -0.0009 & -0.0009 \\
$f_{K \bar{K}}, \mathrm{GeV}^{-1}$ & -0.305 & -0.34 & -0.51 & -0.51 \\
$f_{\pi \eta^{\prime}}, \mathrm{GeV}^{-1}$ & 1.0 & 1.0 & 27.0 & 27.0 \\
$\delta,{ }^{\circ}$ & -77.3 & -67.8 & -94.5 & -94.5 \\
\hline \hline
\end{tabular}

[1] C. Patrignani et al. (Particle Data Group), Chin. Phys. C 40, 100001 (2016).

[2] R. L. Jaffe, Phys. Rev. D 15, 267 (1977); 15, 281 (1977).

[3] N. N. Achasov, S. A. Devyanin, and G. N. Shestakov, Phys. Lett. 108B, 134 (1982); Z. Phys. C 16, 55 (1982).

[4] N. N. Achasov and G. N. Shestakov, Z. Phys. C 41, 309 (1988).

[5] N. N. Achasov and G. N. Shestakov, Phys. Rev. D 77, 074020 (2008); 81, 094029 (2010); Usp. Fiz. Nauk 54, 799 (2011) [Sov. Phys. Usp. 181, 827 (2011)].

[6] N. N. Achasov, Nucl. Phys. A728, 425 (2003).
[7] N. N. Achasov and V. V. Gubin, Phys. Rev. D 63, 094007 (2001).

[8] N. N. Achasov and A. V. Kiselev, Phys. Rev. D 68, 014006 (2003).

[9] N. N. Achasov and V. N. Ivanchenko, Nucl. Phys. B315, 465 (1989).

[10] N. N. Achasov and V. V. Gubin, Phys. Rev. D 56, 4084 (1997).

[11] M. N. Achasov et al. (SND Collaboration), Phys. Lett. B 438, 441 (1998); M. N. Achasov et al., Phys. Lett. B 479, 53 (2000). 
[12] M. N. Achasov et al. (SND Collaboration), Phys. Lett. B 440, 442 (1998); M. N. Achasov et al., Phys. Lett. B 485, 349 (2000); R. R. Akhmetshin et al. (CMD-2 Collaboration), Phys. Lett. B 462, 380 (1999); A. Aloisio et al. (KLOE Collaboration), Phys. Lett. B 537, 21 (2002); C. Bini, P. Gauzzi, S. Giovanella, D. Leone, and S. Miscetti, KLOE Note 173 06/02, http://www.lnf.infn.it/kloe/.

[13] A. Aloisio et al. (KLOE Collaboration), Phys. Lett. B 536, 209 (2002).

[14] N. N. Achasov, V. V. Gubin, and V. I. Shevchenko, Phys. Rev. D 56, 203 (1997).

[15] N. N. Achasov and A. V. Kiselev, Phys. Rev. D 76, 077501 (2007); 78, 058502 (2008).

[16] N. N. Achasov and G. N. Shestakov, Phys. Rev. D 58, 054011 (1998).

[17] N. N. Achasov and A. V. Kiselev, Phys. Rev. D 86, 114010 (2012).

[18] N. N. Achasov and A. V. Kiselev, Int. J. Mod. Phys. Conf. Ser. 35, 1460447 (2014), http://www.worldscientific.com/ doi/pdf/10.1142/S2010194514604475.

[19] N. N. Achasov and G. N. Shestakov, Phys. Rev. D 49, 5779 (1994).

[20] N. N. Achasov and G. N. Shestakov, Phys. Rev. Lett. 99, 072001 (2007).

[21] M. Gell-Mann and M. Levy, Nuovo Cimento 16, 705 (1960).

[22] N. N. Achasov and A. V. Kiselev, Phys. Rev. D 83, 054008 (2011); 85, 094016 (2012).

[23] I. Caprini, G. Colangelo, and H. Leutwyler, Phys. Rev. Lett. 96, 132001 (2006).

[24] S. Acharya et al. (ALICE Collaboration), Phys. Lett. B 774, 64 (2017).

[25] A. Martin, E. Ozmutlu, and E. Squires, Nucl. Phys. B121, 514 (1977).

[26] B. I. Abelev et al. (STAR Collaboration), Phys. Rev. C 74, 054902 (2006).
[27] N. N. Achasov, A. V. Kiselev, and G. N. Shestakov, Phys. At. Nucl. 79, 397 (2016).

[28] R. Lednicky and V. Lyuboshits, Yad. Fiz. 35, 1316 (1982) [Sov. J. Nucl. Phys. 35, 770 (1982)].

[29] A citation from Ref. [26]: "The parameter $\lambda$ measures the correlation strength. In the absence of FSI, $\lambda$ equals unity for a fully chaotic Gaussian source, up to a suppression due to the kaon impurity and finite momentum resolution. Theoretically, it can be less than unity due to partial coherence of the kaon field, resonance decays and the nonGaussian form of the correlation function. Also neglecting FSI can affect (suppress or enhance) the value of this parameter." A citation from Ref. [30]: "In $\pi \pi$ studies the $\lambda$ smaller than 1 has been shown at least in part to be due to the presence of long-lived meson resonances which distort the shape of the source so that the Gaussian assumption, which the fitting functions are based on, is less valid [17]." See also R. Lednicky, Nucl. Phys. A774, 189 (2006) and references therein for details, including the list of assumptions providing the approach.

[30] B. Abelev et al. (ALICE Collaboration), Phys. Lett. B 717, 151 (2012).

[31] B. Abelev et al. (ALICE Collaboration), Phys. Rev. D 87, 052016 (2013); J. Adam et al. (ALICE Collaboration), Phys. Rev. C 92, 054908 (2015).

[32] S. M. Flatté, Phys. Lett. 63B, 224 (1976).

[33] N. N. Achasov and V. V. Gubin, Phys. Lett. B 363, 106 (1995).

[34] N. N. Achasov and A. V. Kiselev, Phys. Rev. D 70, 111901(R) (2004).

[35] N. N. Achasov and G. N. Shestakov, Phys. Rev. D 96, 091501 (2017).

[36] N. N. Achasov, S. A. Devyanin, and G. N. Shestakov, Phys. Lett. 88B, 367 (1979).

[37] S. Uehara et al. (Belle Collaboration), Phys. Rev. D 80, 032001 (2009). 